\title{
Proyecto cubiertas mosaico. Hagamos sostenibles nuestras cubiertas Barcelona + sostenible: proyectos por el clima
}

\section{Mosaic roofs project. Let's make our roofs sustainable Barcelona + sustainable: projects for the climate}

DOI: $10.46932 / \mathrm{sfjdv2n4-093}$

Received in: March 1st, 2021

Accepted in: May 30th, 2021

\author{
Lluïsa Arranz Diez \\ Máster "Laboratorio vivienda siglo XXI", ETSAB \\ Profesional liberal \\ Ingeniera de la Edificación y Diseñadora de Interiores \\ E-mil: LluisaA10@apabcn.cat
}

\section{COMPROMISO DE BARCELONA POR EL CLIMA}

Con motivo de la celebración en París de la $21^{\text {a }}$ Conferencia, de la Convención Marco de las Naciones Unidas sobre el Cambio Climático, donde se reúnen los 196 Estados que la han ratificado, para conseguir un nuevo acuerdo internacional aplicable a todos los países, con el objetivo de mantener el calentamiento global por debajo de $\operatorname{los} 2^{\circ} \mathrm{C}, 800$ entidades ciudadanas vinculadas a la red $\mathrm{B}+\mathrm{S}$, firman el Compromiso Ciudadano por la Sostenibilidad 2012-2022 con el Ayuntamiento de Bcn.

En el otoño de 2015, 9 equipos que han trabajado 9 proyectos, elaboran un documento marco, el "Compromiso de Bcn por el Clima", incluyendo una primera hoja de ruta para el 2015-2017, con la acción colectiva de conseguir que, para el año 2030, Bcn haya reducido un $40 \%$ sus emisiones de $\mathrm{CO}_{2}$, aumentando $1 \mathrm{~m}^{2}$ de verde urbano por habitante.

Cubierta ajardinada

\begin{abstract}
"Se considera una de las mejores soluciones para el medio ambiente.
Utiliza materiales naturales vegetales y tiene valores añadidos. En relación con su comportamiento, humedece el ambiente y hace de barrera en cuanto a la transmisión de las ganancias térmicas solares hacia el interior.

Adicionalmente, se puede utilizar para drenaje de las aguas grises producidas en el edificio. También participa de la calidad del paisaje urbano. El punto débil a controlar en este caso es el consumo de agua." 2
\end{abstract}

Los nuevos pulmones de la ciudad

“Para mitigar el 'efecto de la isla de calor' en las ciudades, se necesita un 10\% más de vegetación.

\footnotetext{
${ }^{1}$ Ayuntamiento de Barcelona (2015). Compromiso de Barcelona por el clima 2015-2017. www.ajuntament.barcelona.cat/premsa/wp-content/uploads/2015/11/Compromis_Bcn_Clima.pdf

${ }^{2}$ Rovira Fontanals, Josep Lluis; Casado Martínez, Imma. Dirección General de la Vivienda, la Arquitectura y el Urbanismo (1999). Guía de la edificación sostenible. Barcelona. Institut Cerdà. Serie: Informes IDAE.
} 
Un $\mathrm{m}^{2}$ de cubierta vegetal absorbe la misma cantidad de $\mathrm{CO}_{2}$ que emitiría un vehículo normal en un recorrido de $80 \mathrm{~km}$.

Las cubiertas verdes mejoran el aislamiento acústico hasta en 8 dB." 3

Huertos en azotea

"Investigadores del Instituto de Ciencia y Tecnología Ambientales (ICTA) de la UAB, en colaboración con el Departamento de Ciencias Agrarias de la Universidad de Bolonia, analizan los impactos ambientales y los costes económicos de la producción de alimentos en huertos comunitarios en azotea desde una perspectiva cuantitativa.

Los resultados aportan nuevos datos y conocimiento sobre la contribución a la sostenibilidad de la agricultura urbana en el marco del diseño urbano. El estudio identifica las técnicas, los cultivos y las prácticas más eco-eficientes, es decir, con un menor impacto ambiental y coste económico." ${ }^{4}$

\section{CUBIERTAS Y MUROS VERDES EN BARCELONA}

En mayo de 2010, el Ayuntamiento de Barcelona en colaboración con la Agencia de Ecología Urbana, publica un extenso estudio sobre las cubiertas y muros verdes existentes, su potencial y estrategias de implantación. ${ }^{5}$

En diciembre de 2015, edita la Guía de tejados vivos y cubiertas verdes. ${ }^{6}$

El 3 de octubre de 2014, el Consejo Municipal, dicta una Medida de Gobierno para impulsar tejados vivos y cubiertas verdes en Barcelona. ${ }^{7}$

Proyecto Cubiertas Mosaico. Hagamos sostenibles nuestras cubiertas

El 31/05/2016, entidades colaboradoras en el proyecto B+S, presentamos el proyecto piloto de Cubiertas Mosaico. ${ }^{8}$

Se trata de una campaña de aprovechamiento de los usos de las azoteas, para generar energía, fomentar el autoconsumo y mejorar la envolvente térmica. Implantar cubiertas verdes y agrícolas, impulsar la producción de energía, el aprovechamiento de las aguas pluviales y generar espacios de disfrute y socialización, en tres tipos de edificios, (administración, zona industrial y comunidad de vecinos/as).

\footnotetext{
${ }^{3}$ Boyé, Josep (Arquitecto Técnico. Resp. Técnico en Sostenibilidad de Knauf Insulation) (2016). Artículo $n^{\circ}$ 127. Cubiertas verdes ligeras. Madrid. Cercha. www.knaufinsulation.es/sites/ki_es/files/uploads/CERCHA_1.pdf

${ }^{4}$ Instituto de Ciencia y Tecnología Ambientales (ICTA) de la UAB, en colaboración con el Departamento de Ciencias Agrarias de la Universidad de Bolonia. La selección de técnicas y cultivos en azotea (2016). https://www.uab.cat/web/detallenoticia/la-seleccion-de-tecnicas-y-cultivos-puede-determinar-el-impacto-ambiental-y-economico-de-los-huertos-en-azotea1345680342040.html? noticiaid $=1345697906608$

5 Ayuntamiento de Barcelona en colaboración con la Agencia de Ecología Urbana (2010). Cubiertas y Muros Verdes en Barcelona. http://bcnecologia.net/sites/default/files/proyectos/doc_cobertes_i_murs_verds_01m2010_0.pdf

6 Ayuntamiento de Barcelona (2015). Guía de tejados vivos y cubiertas verdes. https://bcnroc.ajuntament.barcelona.cat/jspui/bitstream/11703/86542/7/Guia_Terrats_i_Cobertes_Verdes.pdf

${ }^{7}$ Consejo Municipal de Barcelona (2014). Medida de Gobierno para impulsar terrados vivos y cubiertas verdes en Barcelona. https://bcnroc.ajuntament.barcelona.cat/jspui/bitstream/11703/84720/1/14168.pdf

${ }^{8}$ Entidades colaboradoras en el proyecto B+S (2016). Proyecto Cubiertas Mosaico. Hagamos sostenibles nuestras cubiertas. www.lameva.barcelona.cat/barcelonasostenible/sites/default/files/pagines/document/5535/posterscbpcprojectescobertes.pdf
} 


\section{FASES DEL PROYECTO}

0. Establecer un equipo de trabajo interdisciplinar (agua, alimentación, verde, energía).

1. Encontrar un ejemplo de cada edificio tipo.

2. Edificios cercanos para visualizar el mosaico.

3. Estudio de viabilidad.

4. Seguimiento mediante indicadores.

5. Comercializar la producción agrícola. Mercados de proximidad.

6. Mantenimiento.

7. Balance social, energético, económico, $\mathrm{CO}_{2}$. Replicabilidad.

8. Comunicación/Educación.

Fachadas, cubiertas verdes y azoteas vivas

El 7 de octubre de 2016, dentro de la Semana de la Rehabilitación (REHABILITA) en el CAATEEB se realiza una presentación. ${ }^{9}$

"La diferencia de temperatura entre la ciudad y el campo o espacio periurbano que la rodea se conoce como 'efecto isla de calor'. En verano, esta diferencia térmica puede llegar casi a $10^{\circ} \mathrm{C}$ y provocar una disminución de la calidad de vida y salud de los habitantes de las ciudades.

Los espacios verdes de las ciudades y las fachadas y cubiertas verdes de los edificios colaboran en la disminución de este calentamiento, siendo una buena solución ya que reducen el efecto isla de calor a través del proceso de transpiración y humidificación del aire seco, que mejoran el clima y aumentan la sensación de bienestar. También pueden ser una solución para la captación y almacenamiento de agua, reducción de los niveles de contaminación, creación de nuevos hábitats para la fauna, mejora del paisaje urbano y de la calidad de vida, potenciación de la agricultura ecológica, etc."

\section{RESULTADOS Y EVALUACIÓN DE LOS PROYECTOS POR EL CLIMA}

El 13 de diciembre de 2017, 14 entidades participantes en la red B+S compartimos la experiencia de nuestros proyectos. ${ }^{10}$

Valoración y nuevas perspectivas para los proyectos por el clima: ${ }^{11}$

“Satisfacción, orgullo por el trabajo bien hecho, aprendizaje, generación de vínculos y percepción del potencial del trabajo conjunto son algunos de los beneficios personales que se destacaron de la implicación en los proyectos. Entre las capacidades y habilidades potenciadas, se mencionó la

\footnotetext{
${ }^{9}$ Semana de la Rehabilitación (REHABILITA) (2016). CAATEEB. www.apabcn.cat/documentacio/areatecnica/PDFS_SHAREPOINT/Presentacions/FA\%C3\%87ANES-VERDES-07-102016/PABLO-NAVARRO.PDF

${ }^{10}$ Participantes en la red $\mathrm{B}+\mathrm{S}$ (2017). Resultados y evaluación de los proyectos por el clima . http://lameva.barcelona.cat/barcelonasostenible/ca/entitats-i-empreses/bulleti/470/valoracio-i-noves-perspectives-per-alsprojectes-pel-clima

${ }^{11}$ Equipos de proyecto del Compromiso de Barcelona por Clima (2017). Informe. Compartimos los resultados y la evaluación de los proyectos por el clima. http://lameva.barcelona.cat/barcelonasostenible/sites/default/files/articles/document/8522/informecompartimelsresultatsilaval uaciodelsprojectespelclima.pdf
} 
habilidad para trabajar en equipos multidisciplinares, la mejora de las capacidades comunicativas y el aprendizaje de nuevas técnicas y metodología.

En cuanto a los factores que pueden bloquear este tipo de proyectos, se habló de falta de recursos económicos, la participación desigual, la distancia entre las expectativas y los resultados, la falta de comunicación inclusiva, momentos de rivalidad y falta de liderazgo. Para mejorar los proyectos en el futuro, se propuso definir los recursos y plantear los resultados esperados desde el inicio. Con carácter general, se considera que la incertidumbre asociada a esta experiencia ha permitido adquirir unos aprendizajes útiles para futuras ediciones."

El grupo Cubiertas Mosaico, después de efectuar diversas acciones de promoción, no ha conseguido impulsar la realización de las tres cubiertas piloto propuestas.

Los principales motivos detectados que impidieron la realización de las tres cubiertas piloto, fueron de carácter económico, la falta de consenso entre los propietarios de las fincas y el desconocimiento de los valores y de las cuestiones que afectan a la construcción y el mantenimiento de las cubiertas verdes.

No obstante, con la campaña "Hagamos sostenibles nuestras azoteas" ${ }^{12}$, ha impulsado y dado a conocer las cubiertas verdes en la ciudad, mediante una hoja informativa e interviniendo en la elaboración de las bases del concurso convocado por el Instituto Municipal del Paisaje Urbano.

\section{CONCURSO DE CUBIERTAS VERDES}

En junio de 2017, el Ayuntamiento de Bcn impulsa el concurso de cubiertas verdes ${ }^{13}$, para promover la implantación de nuevas azoteas verdes en edificios de viviendas, equipamientos docentes, sanitarios o de otros usos. Todos ellos con un fuerte impacto ambiental, social y paisajístico. ${ }^{14}$

Se presentaron 45 proyectos, que disponían de 60 días para elaborar una propuesta técnica con la que evaluar su viabilidad e idoneidad y poder seleccionar los ganadores. Durante esta fase, el Ayuntamiento ofreció un apoyo financiero de $1.500 €$ a los preseleccionados para los trabajos técnicos de implementación de la cubierta.

Finalmente se escogieron 10 propuestas ganadoras que recibirán una subvención del $75 \%$ del coste de las obras y los estudios técnicos necesarios, hasta un límite de $100.000 €$. El 17 de mayo de 2018, el Ayuntamiento de Bcn presenta las propuestas ganadoras del concurso. ${ }^{15}$

La primera cubierta del concurso, Escuelas Salesianas de Sarrià en el Paseo de San Joan Bosco $n^{\circ}$ 42, se inauguró el 26 de noviembre de 2018.

\footnotetext{
${ }^{12}$ Grupo Cubiertas Mosaico (2017). Cubiertas mosaico. Hagamos sostenibles nuestras azoteas. https://www.youtube.com/watch?v=efMDBmvkvug\&index=1\&list=PL-_DKISeIjZk1ZLjv1sj1EwaS5Kvq4nzf

13 Grupo Cubiertas Mosaico (2017). Quien tiene una cubierta tiene un tesoro. Concurso de Cubiertas Verdes. Demos paso al verde. https://mediaedg.barcelona.cat/wp-content/uploads/2017/07/27131553/Triptic-Cobertes-Verdes.pdf

14 Grupo Cubiertas Mosaico (2017). Quien tiene una cubierta tiene un tesoro. Concurso de Cubiertas Verdes. Tienes dudas sobre las cubiertas verdes? https://ajuntament.barcelona.cat/ecologiaurbana/sites/default/files/FaqsCobertesVerdes.pdf

15 Ayuntamiento de Barcelona $\left({ }^{2018}\right)$. Propuestas ganadoras del concurso de Cubiertas Verdes. http://ajuntament.barcelona.cat/premsa/wpcontent/uploads/2018/05/10propostesguanyadorescobertes.pdf
} 
El conjunto edificado cierra perimetralmente una gran isla que en el interior dispone de dos patios situados a distinto nivel, entre los que se sitúa una pérgola en principio no transitable. Se propone hacerla accesible, e implantar una cubierta extensiva con plantas tapizantes, así como arbustivas y enredaderas junto a la fachada, creando un nuevo espacio de estancia. Esta nueva superficie verde se sitúa en un interior de manzana desprovisto actualmente de vegetación, y por tanto tendrá un gran impacto visual desde las aulas.

Se ubican placas fotovoltaicas, que instalan los propios alumnos del ciclo formativo de energías renovables. Además de aprovechar la energía producida, serán un recurso pedagógico en este campo técnico.

Contará con un sistema de riego por goteo con sensores de humedad. Para potenciar la biodiversidad del lugar se instalará un hotel de insectos que ayudará a la polinización de las especies vegetales.

Fig. 1. Proyecto Paseo de Sant Joan Bosco, $n^{\circ} 42$ (Sarrià-Sant Gervasi)

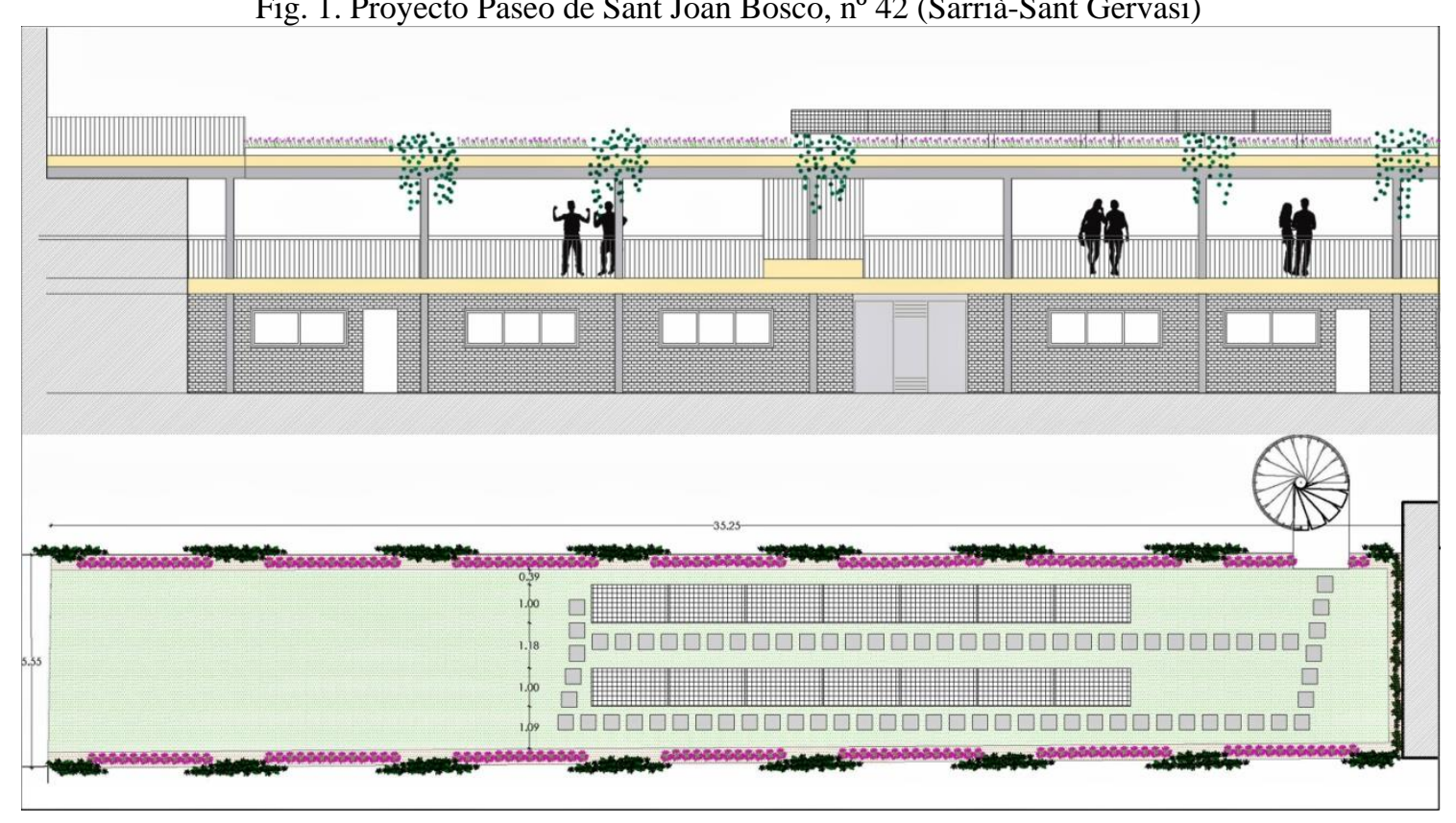


Fig. 2. Cubierta verde en el Paseo de Sant Joan Bosco, nº 42 (Sarrià-Sant Gervasi)

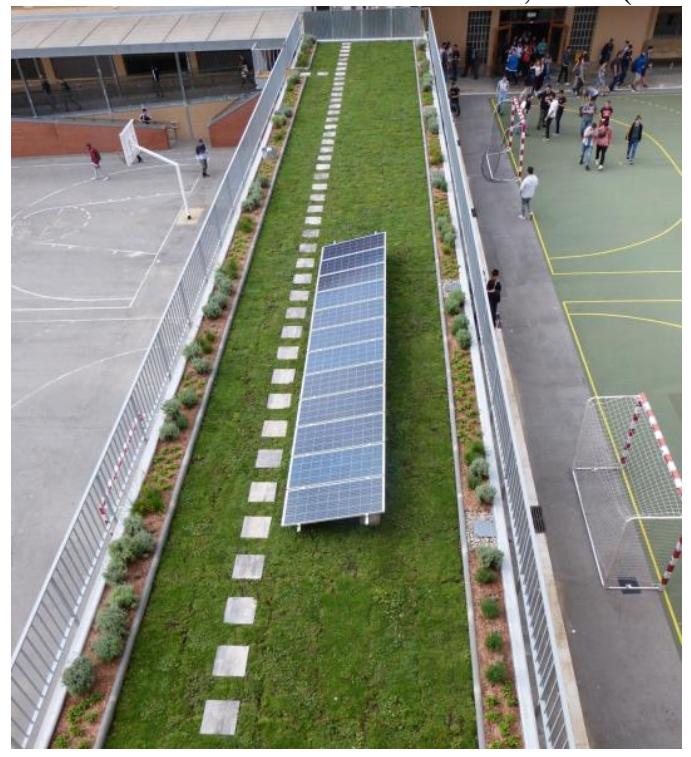

La cubierta verde de la Calle de Balcells n 41, se comenzó a construir en noviembre de 2018.

A partir de la experiencia desarrollada en el jardín de la parcela, la Comunidad de Vecinos propone una azotea donde los cultivos y la biodiversidad se potencien. Se generan espacios con diversas especies hortícolas y tablas de cultivo aprovechando el abono generado en las compostadoras y el agua de lluvia que se recoge en un depósito en la cubierta. También se prevé plantar aromáticas y arbustos para crear una protección contra el viento, así como enredaderas en los muros.

Un pequeño estanque de peces ayuda a la fertilización de un área de cultivo acuapónico, donde se cultivarán verduras de hoja. Un jardín con plantas mediterráneas, susceptibles de ser refugio de insectos, se plantea como jardín de mariposas, que junto a un hotel de insectos facilitan la polinización de los huertos. También se implantan nidos y comederos para aves.

La selección de plantas, más el sombreado y el acolchado reducirán las necesidades hídricas, que se nutrirán de agua de lluvia distribuida con un sistema por goteo, cuya bomba utilizará la energía producida por la nueva instalación fotovoltaica. 
Fig. 3. Y Fig. 4. Proyecto Calle de Balcells nº 41 (Gràcia)
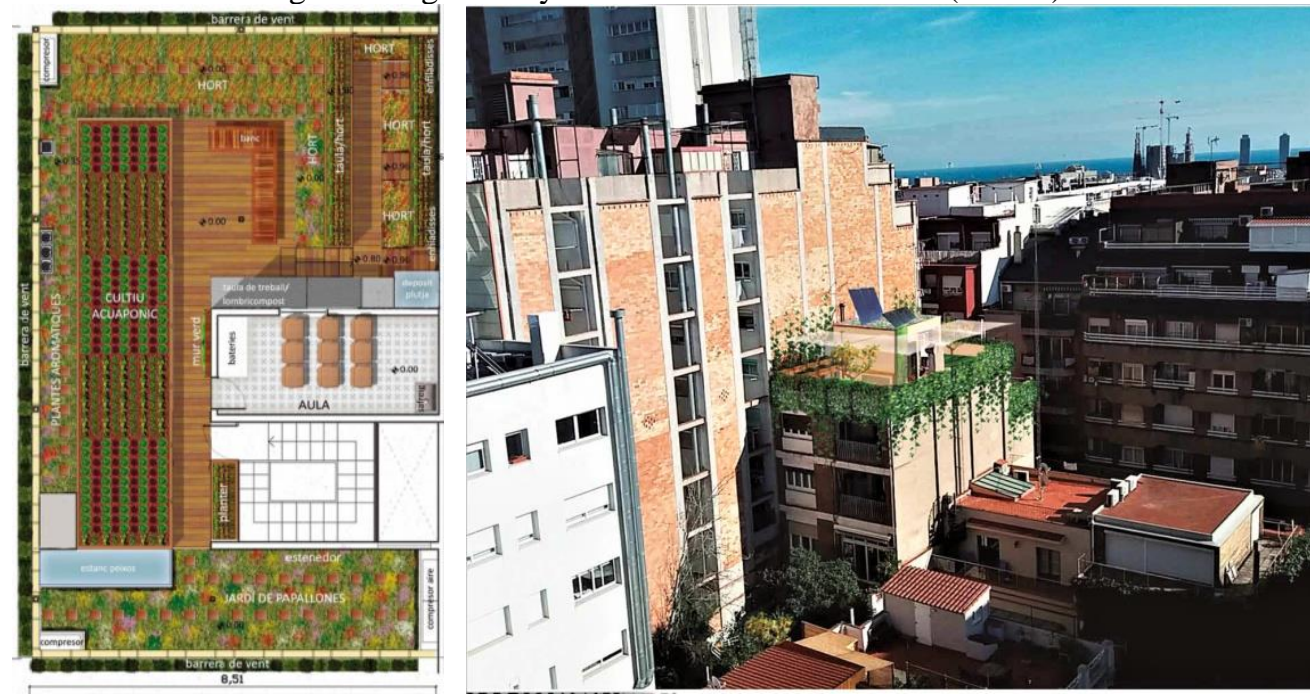

En enero de 2019 comenzaron las obras en la Calle de Aragón nº 174.

Situada en el encuentro de las calles de Aragón y Casanovas, la edificación está formada por torres con 133 viviendas y un zócalo de tres plantas, con 15 locales y aparcamiento. Se propone ajardinar la cubierta de este último volumen situado en el interior de la manzana. Por lo tanto, podrán acceder todos los usuarios de la finca y podrán disfrutarlo los vecinos de las viviendas que tienen vistas sobre el interior de la manzana.

Se propone plantación de sedums, plantas aromáticas y gramíneas. Las zonas ajardinadas alternan con pasos pavimentados y zonas con grava. Se abre un nuevo acceso directo a esta azotea para facilitar su uso a los vecinos con movilidad reducida. Se prevé instalar un hotel de insectos para mejorar la biodiversidad.

El sistema de riego por goteo enterrado, permite optimizar el consumo de agua, que en parte provendrá de lluvia retenida en el sustrato.

Fig. 5. Proyecto Calle de Aragón no 174 (Eixample)

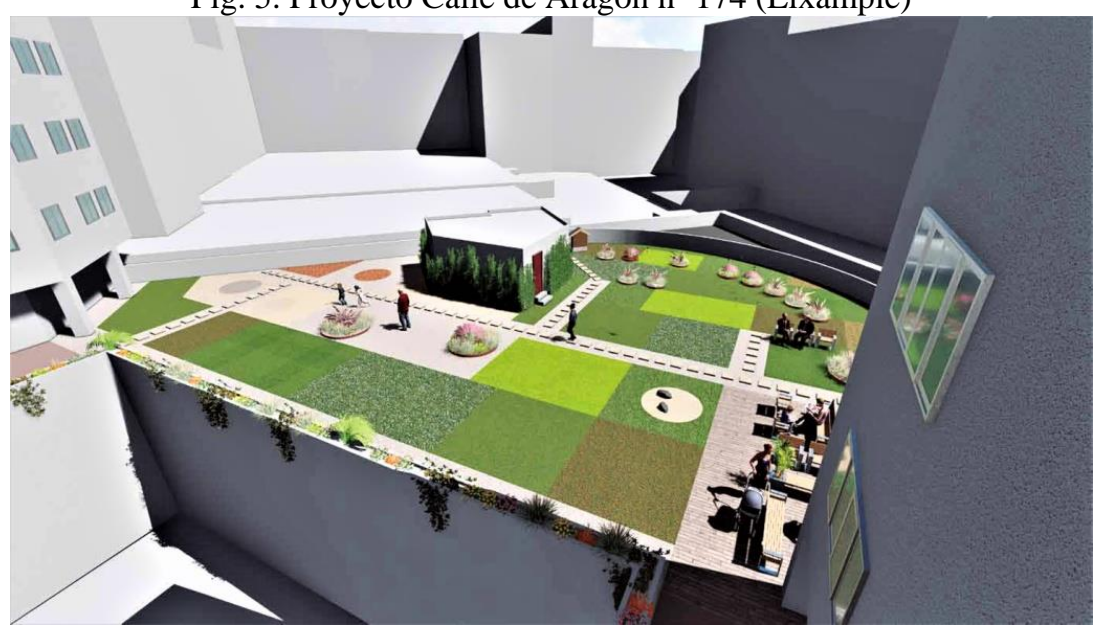


Fig. 6. Calle de Aragón nº 174 (Eixample)

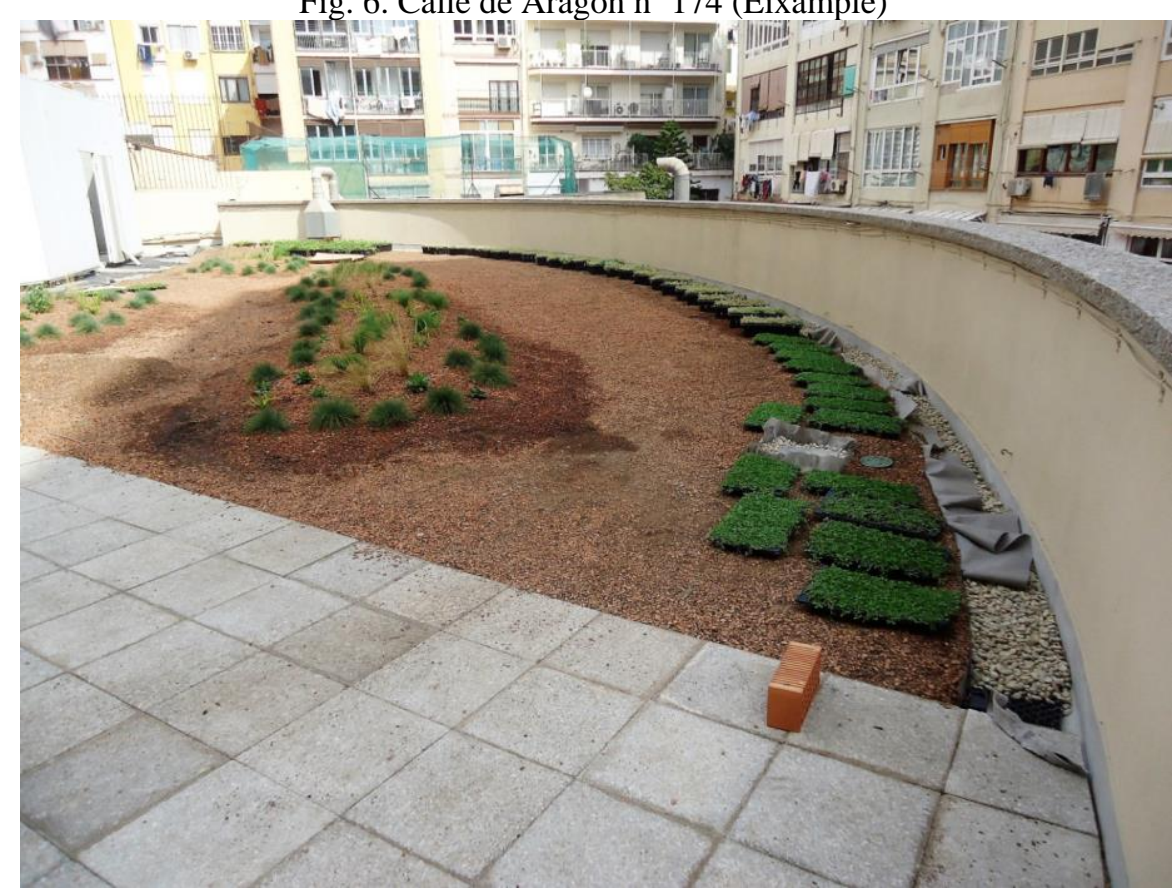

\section{EXPOSICIÓN Y COLOQUIO}

En el CCCB, el 8 de febrero de 2018, la red de firmantes del Compromiso Ciudadano, organizan una exposición "Después del fin del mundo visto por $\mathrm{B}+\mathrm{S}$ "16, visita con los equipos implicados y el coloquio "Las cubiertas verdes, elementos clave para conseguir una ciudad más verde y sostenible" ${ }^{17}$.

\footnotetext{
16 Red de firmantes del Compromiso Ciudadano (2018). Exposición. Después del fin del mundo visto por B+S. http://www.cccb.org/ca/activitats/fitxa/despres-de-la-fi-del-mon-vist-per-barcelona-sostenible/227700

${ }^{17}$ Red de firmantes del Compromiso Ciudadano (2018). Coloquio. Las cubiertas verdes, elementos clave para conseguir una ciudad más verde y sostenible.

http://www.cccb.org/es/actividades/ficha/las-cubiertas-verdes-elementos-clave-para-conseguir-una-ciudad-mas-verde-ysostenible/227639
} 
Fig. 7. Después del fin del mundo visto por $\mathrm{B}+\mathrm{S}$

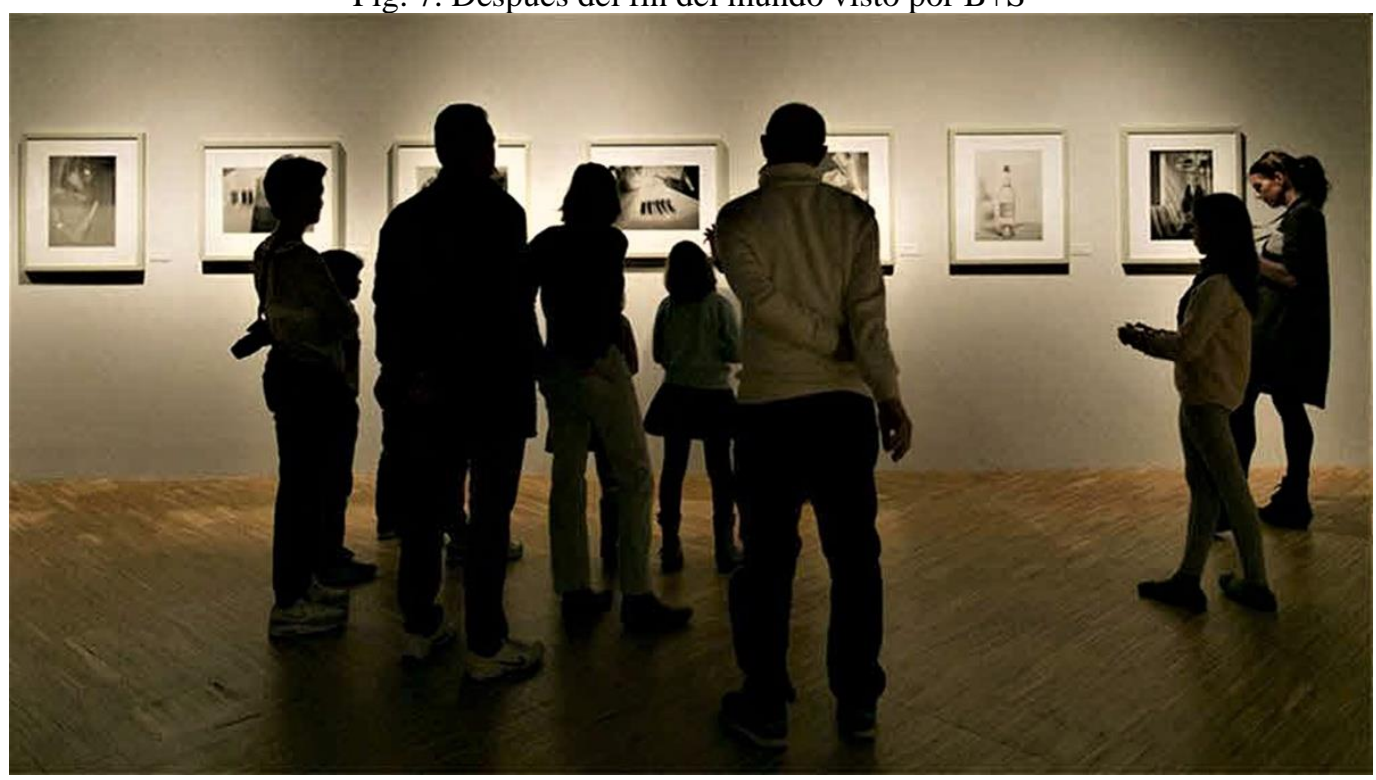

7 PROYECTO EN EL MARCO DE LAS AYUDAS DEL COMPROMISO DE BCN POR EL CLIMA

"Barreras y Oportunidades de las Cubiertas Mosaico"

Miembros del grupo de trabajo Cubiertas Mosaico queremos aprovechar el empuje del concurso y recoger, gestionar, analizar y publicar datos clave, mediante seminarios con los grupos de interés en relación con los proyectos seleccionados y que se están implantando a partir de las subvenciones otorgadas, (técnicos, vecinos, presidentes de comunidad, administradores de fincas...).

Este proyecto quiere analizar el proceso de implantación de las cubiertas seleccionadas, desde los puntos de vista legal, tecnológico, arquitectónico, ambiental, económico y social. Detectar las barreras en el proceso de diseño y ejecución de las propuestas. Proponer y aplicar indicadores cualitativos y cuantitativos de seguimiento en los aspectos energéticos, hídricos, verde y alimentos y proponer acciones de mejora para facilitar la implantación de futuras cubiertas verdes. Se editará un documento de difusión de los resultados del estudio en formato digital.

Contribución a los resultados de los objetivos del Compromiso por el Clima

El proyecto se alinea con Objetivos del Plan por el Clima 2018-2030:

- L3: Prevenir el calor.

Intervenir sobre pavimentos y azoteas para incrementar el índice de reflectancia.

- L5: Recuperar las Azoteas.

Consolidar el concurso de Cubiertas Verdes (anualmente). 
- L6: Planificar en clave climática.

Reservar espacio en el suelo, el subsuelo y la azotea, para la prestación de servicios climáticos.

- L7: Mucho más verde.

Planificar de forma participativa la red de corredores verdes urbanos. Crear jardines y una red de reservas de naturaleza urbana.

- L8: Ni una gota perdida.

Aumentar la permeabilidad del suelo a través de una estrategia de drenaje urbano sostenible.

- L16: Acción cultural por el clima.

Disponer de un referente de sostenibilidad en cada distrito.

Fig. 8. Seminario Barreras y Oportunidades de las Cubiertas Mosaico

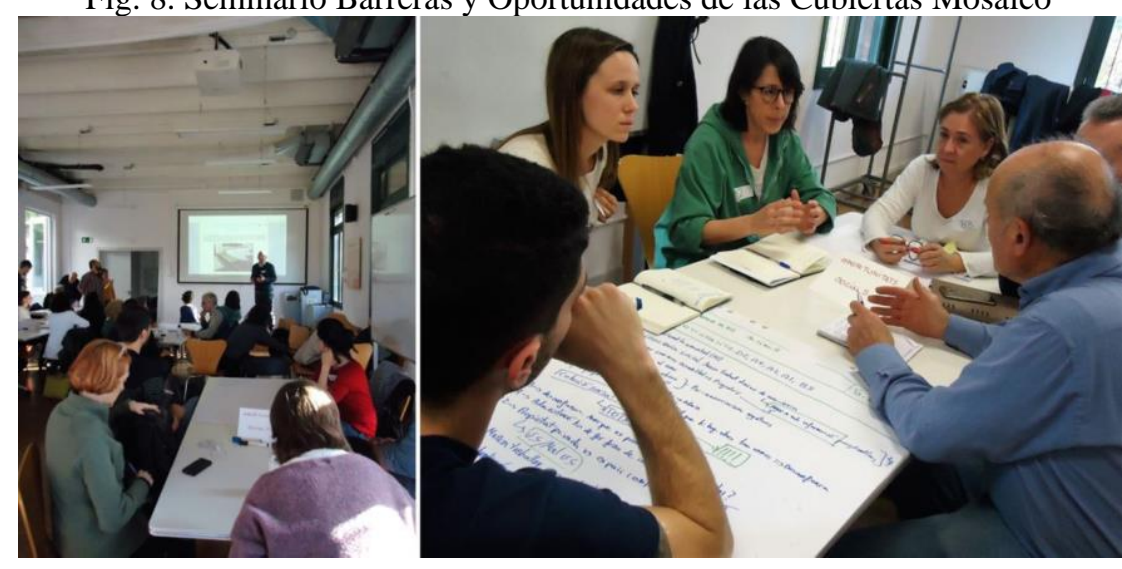

Integrantes del grupo

- EixVerd (Lidia Calvo, Ingeniera). Empresa de diseño, ejecución y mantenimiento de soluciones para adaptar la ciudad al cambio climático. Con un equipo de cuatro personas entre los cuales, un arquitecto, un arquitecto técnico y una jardinera.

- ICTA-UAB. Instituto de Ciencia y Tecnología Ambiental (Dr. Joan Rieradevall). Investigador del grupo Sostenipra. Ha publicado más de 140 artículos en revistas indexadas y unos 20 libros de temas ambientales asociados a la energía, el agua y los alimentos en ciudades. Investigadores responsables: Dr. Xavier Gabarrell y Susana Toboso, Máster Universitaria en Ciencias.

- 4A+A Arquitectura Ambiental (Julio Bermejo, Arquitecto). Despacho de arquitectura, y consultoría ambiental y energética. Conformado por tres socios, se fundó en el año 2011 como estudio de arquitectura sostenible, con especialización en obra nueva y rehabilitación energética integral de edificios. 
- COAC-Aus (Dra. Alrun Jimeno). Agrupación del COAC. Ha colaborado en las bases del concurso "Cubiertas Mosaico". En 2008 en la EPSEVG de Vilanova y la Geltrú, con compañeros de la UPC, proyectó sustituir $2.000 \mathrm{~m}^{2}$ de cubierta inclinada de fibrocemento por una plana transitable, preparada para captar energías renovables y permitir plantación vegetal.

- ENTORN XXI. Educación ambiental, compostaje doméstico, calidad ambiental, gestión de espacios naturales, estudios de impacto ambiental y rehabilitación de viviendas. (Lluïsa Arranz, Ingeniera de Edificación). Máster en Laboratorio de la Vivienda Sostenible del Siglo XXI. Graduada Superior en Diseño de Interiores. Ponencias: $5^{\circ}$ Congreso de Patología y Rehabilitación de Edificios, FEUP 2015; $1^{\circ}$ Congreso Internacional de Vivienda Colectiva Sostenible, ETSAB 2014. Miembro de UNESCO Garraf. 